\title{
Metal Area Segmentation in X-ray CT Images Using the RNA (Relevant Neighbor Area) Principle
}

\author{
Youngshin $\mathrm{Kim}^{\dagger}$, Hyukjoon $\mathrm{Kwon}^{++}$, Joongkyu $\mathrm{Kim}^{+++}$, Juneho $\mathrm{Yi}^{++++}$
}

\begin{abstract}
The problem of Metal Area Segmentation (MAS) in X-ray CT images is a very hard task because of metal artifacts. This research features a practical yet effective method for MAS in X-ray CT images that exploits both projection image and reconstructed image spaces. We employ the Relevant Neighbor Area (RNA) idea [1] originally developed for projection image inpainting in order to create a novel feature in the projection image space that distinctively represents metal and near-metal pixels with opposite signs. In the reconstructed result of the feature image, application of a simple thresholding technique provides accurate segmentation of metal areas due to nice separation of near-metal areas from metal areas in its histogram.
\end{abstract}

Key words: Medical Imaging, X-ray CT, Metal Area Segmentation, Relevant Neighbor Area, Metal Artifacts Reduction

\section{INTRODUCTION}

Metal Area Segmentation (MAS) research in $\mathrm{X}$-ray CT images has been pursued in the context of solving the problem of Metal Artifact Reduction (MAR). In most MAR work, a solution to MAS is necessary in order to effectively remove metal artifacts caused by dense objects such as metal.

※ Corresponding Author: Juneho Yi, Address: (440746) 300 Chunchun-dong Jangan-gu, Suwon, Korea, TEL : +82-31-290-7142, FAX : +82-31-290-7947, E-mail : jhyi@skku.edu

Receipt date: Sep. 16, 2012, Revision date : Oct. 19, 2012

Approval date: Nov. 13, 2012

' School of Electronic and Electrical Computer Engineering Sungkyunkwan University, Korea

(E-mail: karisdo@gmail.com)

${ }^{++}$School of Electronic and Electrical Computer Engineering Sungkyunkwan University, Korea (E-mail: lunlun@skku.edu)

${ }^{+++}$School of Electronic and Electrical Computer Engineering Sungkyunkwan University, Korea (E-mail: jkkim@skku.edu)

${ }^{++++}$School of Electronic and Electrical Computer Engineering Sungkyunkwan University, Korea

※ This work was supported in part by Minstry of Knowledge and Economy, grant 10031993, and by the National Research Foundation of Korea (NRF), grant 2011-0006637.
MAS in X-ray CT images can be carried out either in the projection image space or in reconstructed image space. Henceforth, we refer to the former approach as Projection Image Segmentation (PIS) and the latter as Reconstructed Image Segmentation (RIS). Both PIS and RIS have their own drawbacks. Since metal information in a projection image gets dispersed, MAS in the projection image space is inherently hard. RIS is relatively easy compared to PIS in that metal area appears spatially concentrated in reconstructed images, but still suffers from artifacts near metal.

In this research, we propose a simple yet effective hybrid segmentation (HS) method that overcomes the drawbacks of PIS and RIS by exploiting both image spaces. As can be seen in Fig. 1, we first transform the projection image so that metal, near metal, and non-metal areas are more distinctively represented in the transformed image. We call this image 'pseudoPIS'. In order to create pseudoPIS, we exploit the Relevant Neighbor Area (RNA) principle [1] that was originally developed for projection image inpainting to boost the MAR performance. The RNA principle can simply be 


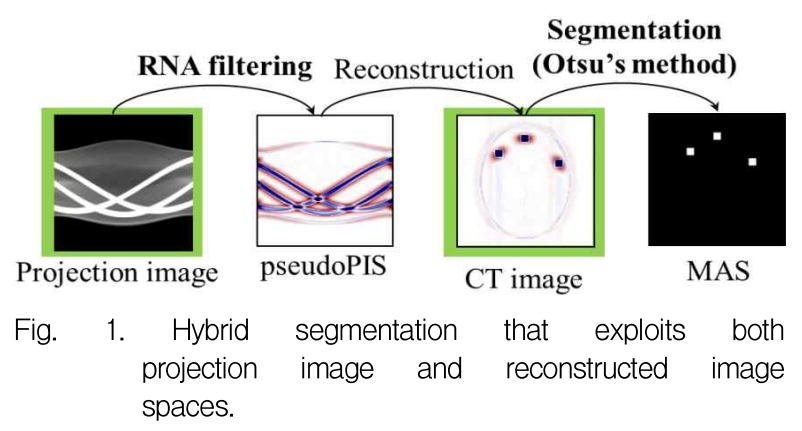

stated as "In the absence of highly dense object such as metal, a pixel in projection image is well represented by a weighted average of its neighbor pixels in RNA". The RNA signifies neighbor pixels that have information relevant to the target pixel to be inpainted. The pixels and their weights in a RNA are computed by forward-projecting the area that affects the target pixel to be inpainted.

We basically find outlier pixels in the projection image that do not follow the RNA principle since those outlier pixels are likely to correspond to metal and possibly, near-metal areas. This process yields a transformed projection image, 'pseudoPIS'. In pseudoPIS, metal and near-metal pixels are distinctively represented by opposite signs with some magnitude and non-metal pixels show small values close to zero. The distinction between metal and near-metal pixels carries over to the reconstructed image of pseudoPIS. In the reconstructed image of pseudoPIS, we exclude pixels belonging to near-metal and simply distinguish between metal and non-metal pixels using histogram thresholding. We have used the Otsu's method [2] where an optimal threshold value is found such that the maximum ratio of between-class to within-class scatter is achieved. However, a variant method [3] using the Otsu's method can also be employed. Experimental results show that the performance of the proposed hybrid segmentation method outperforms that of PIS and RIS.

\section{RELATED WORK}

As previously mentioned, researches of MAS in
X-ray CT images can be divided into PIS and RIS. In the category of PIS, a Markov random field (MRF) model in combination with Bayesian techniques is applied to obtain segmentation results [4] In [5], a projection image is treated as an image formed by two regions of approximately piecewise-constant intensities and represented by the active contours model. RIS often achieves MAS by thresholding the initially reconstructed image where metal artifacts are not removed. The segmentation results are fed into the MAR process, irrespective of whether they are FBP (Filter back-projection) based methods [6] or statistical methods [7]. Akhoondali et al. [8] used a region growing method and Zhang et al. [9] employed Laplacian diffusion based automatic metal segmentation.

PIS is inherently hard because metal informations gets dispersed in the projection image space. In contrast, RIS is relatively easier than PIS in the sense that metal pixels are spatially in proximity. However, distinction between metal areas and near-metal areas in the reconstructed image space remains a formidable task because a reconstructed image suffers from metal artifacts near metal.

\section{RNA (RELEVANT NEIGHBOR AREA)}

In this section, we briefly overview the RNA idea [1] that was first proposed as an effective method for projection image inpainting for MAR. The next section describes how we exploit this RNA idea for MAS in X-ray CT images.

The RNA principle can simply stated as "In absence of highly dense object such as metal, a target pixel to be inpainted is well represented as a weighted average of RNA pixels". The RNA signifies neighbor pixels that have information relevant to the target pixel to be inpainted. The RNA idea innovatively reduces inpainting errors simply by excluding the neighbor pixels that have no effect on the target pixel to be inpainted. 


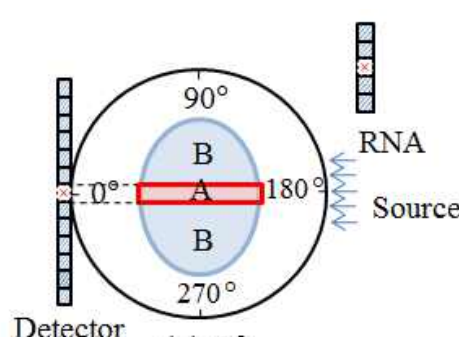

(a) $0^{\circ}$

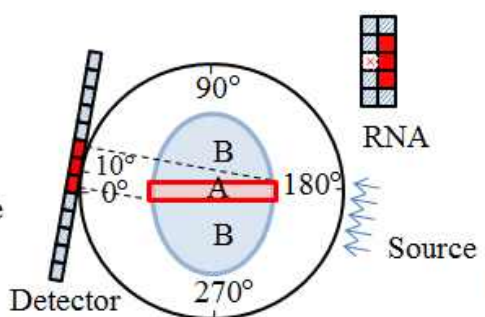

(b) $10^{\circ}$

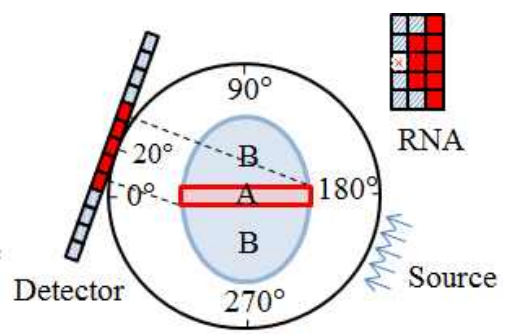

(c) $20^{\circ}$

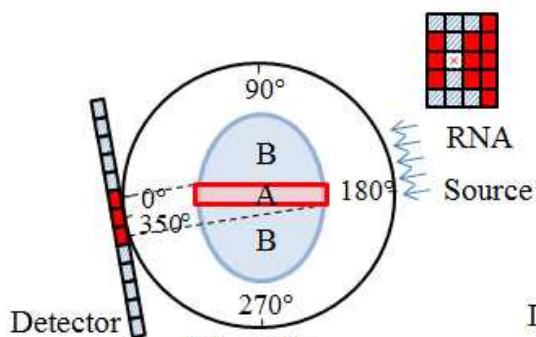

(d) $350^{\circ}$

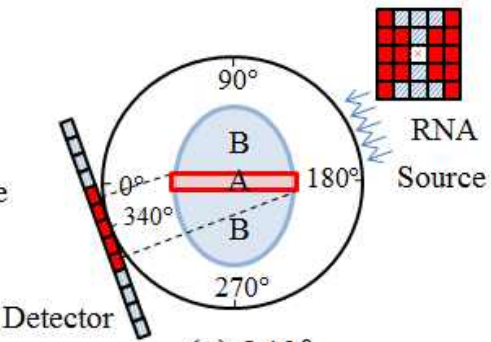

(e) $340^{\circ}$

Fig. 2. A simulated example of forming an RNA in a $5 \times 5$ rectangular neighborhood.

Fig. 2 illustrates how an RNA is formed in the projection image using a simple simulation where the detector array consists of 13 pixels and rotates in $10 \mathrm{deg}$ increment. The projection image is a two-dimensional array whose column contains the value of the detector array at each angle. The value of pixel, $p$, in the projection image depends on the attenuation coefficients (AC) of the object's area through which $\mathrm{X}$-rays pass. That is, the value of the pixel $p$ is only affected by AC's of area A as shown in Fig. 2 (a). When the detector is rotated, the neighbor pixels that get to have $\mathrm{AC}$ information of area A can only affect the inpainting value of the pixel. The complete set of the RNA pixels in case of a $5 \times 5$ rectangular neighborhood for the target pixel, $p$ (marked with ' $\mathrm{x}$ ') to be inpainted is shown in Fig. 2 (e). The pixels and their exact weights in an RNA can be computed via forward-projection of area $\mathrm{A}$.

\section{HYBRID SEGMENTATION USING THE RNA PRINCIPLE}

As previously described, we find outlier pixels in the projection image that do not follow the RNA principle. That is, we do not explicitly label regions in pseudoPIS but only provide salient distinction between metal, near-metal, and non-metal regions so that MAS can be facilitated in the reconstructed image of pseudoPIS.

PseudoPIS is produced by transforming the projection image using equation (1). $I(x, y)$ denotes the intensity value of pixel $(x, y)$ in the projection image and $R$ represents RNA filtering of which the output is the weighted average of pixels in the RNA of pixel $(x, y)$. We simply compute the difference image, $D(x, y, R)$, by subtracting $R(I(x, y))$ from $I(x, y)$ :

$$
D(x, y, R)=I(x, y)-R(I(x, y))
$$

As long as we set the size of RNA so that RNA may not be fully contained in areas corresponding to metal in the projection image, the $D(x, y, R)$ feature space can be divided into three regions $\mathrm{de}^{-}$ pending on the value of $D(x, y, R)$. A pixel value, $D$, close to zero explains that the pixel is likely to belong to non-metal since it obeys the RNA principle that a non-metal pixel is well approximated using the weighted average of its RNA pixels. A pixel with either positive or negative $D$ away from zero can be considered an outlier that does not satisfy the RNA principle. A pixel with positive $D$ away 
from zero is likely to be a pixel in metal area. The RNA region of a pixel in metal area overlaps with non-metal area of which pixel values are smaller than those in metal region. In contrast, a pixel with negative $D$ away from zero is likely to belong to non-metal area near metal. In this case, its corresponding RNA region overlaps with metal area and the weighted average of the RNA pixels gets larger than the value of the pixel considered. In the current implementation, the size of RNA mask is $\mathrm{ex}^{-}$ perimentally decided using various synthetic projection images created with the number of metal pieces and their sizes varied. We are investigating into an effective learning method that computes an optimal size of RNA mask through training.

As previously mentioned, metal artifacts make it hard to distinguish metal pixels from non-metal pixels near metal that possibly belong to an air, tissue or tooth region. Figs 3. (a) and (b) display histograms of the reconstructed images of a projection image and its pseudoPIS, respectively. We have traced down non-metal pixels near metal that belong to air, tissue or tooth regions in order to see where in the histogram they have ended up. We have observed that, in the histogram of the reconstructed image of pseudoPIS, metal and nonmetal pixels near metal get distributed on the opposite sides. This distribution stems from the fact that distinction of metal and non-metal pixels near metal in the histogram of pseudoPIS with opposite signs carries over to the histogram of its $\mathrm{re}^{-}$ constructed image. This enables us to effectively exclude non-metal pixels near metal and facilitates the problem of metal area segmentation. In contrast, in Fig 3. (a) which displays the histogram of a reconstructed image of the projection image without employing the feature from equation (1), non-metal pixels near metal that belong to air, tissue or tooth regions get mixed with metal pixels.

\section{EXPERIMENTAL RESULTS}

For the experiments, we have chosen dental CT images because their readability is poorer than body CT images. Assuming the photon energy of $80 \mathrm{KeV}$ under typical dental CT imaging environment, we have modeled dental CT images with metallic material normally used for dental prosthe ${ }^{-}$ ses such as implants or crown. The mass attenuation coefficients [10] of $\mathrm{Ag}, \mathrm{Ni}, \mathrm{Ti}$, and $\mathrm{Al}$ are used for creating experimental images. A dental CT image with three metallic prostheses is shown in Fig. 4 (a) with its ground truth segmentation in Fig. 4 (b)

Figs. 4 (c), (d), and (e) present the segmentation results of PIS, RIS and HS (hybrid segmentation), respectively, in the reconstructed image space. We have chosen histogram thresholding for segmentation in order to show the effectiveness of the $D(x$, $y, R)$ feature that is computed from the RNA

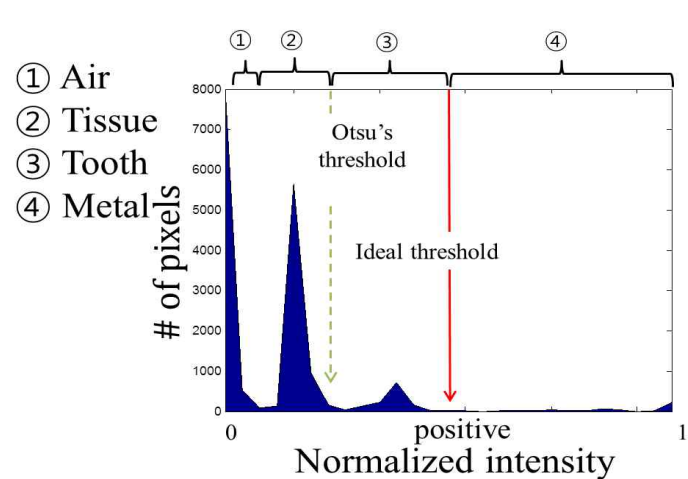

(a)

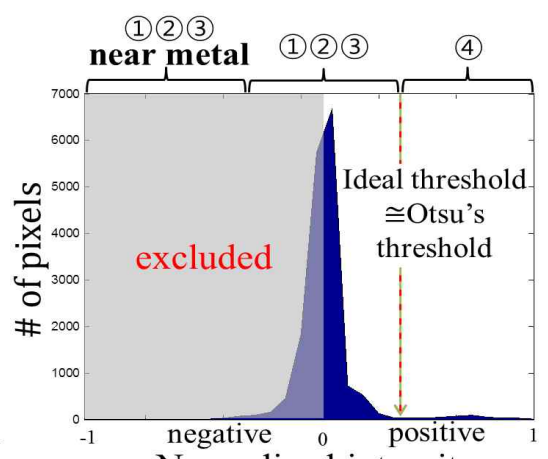

(b)

Fig. 3. Comparison of histograms: (a) Reconstructed image of projection image, (b) Reconstructed image of pseudoPIS. 


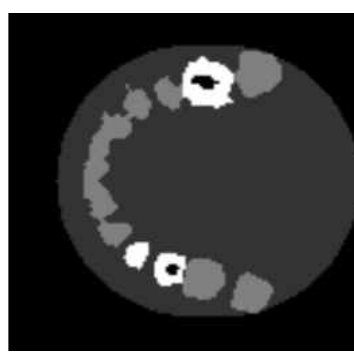

(a) Original CT image

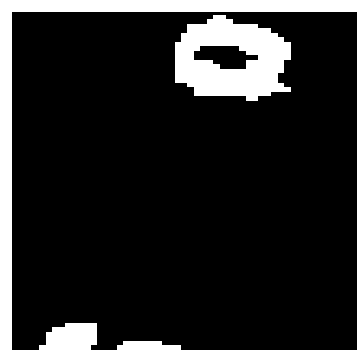

(b) MAS (ground truth)

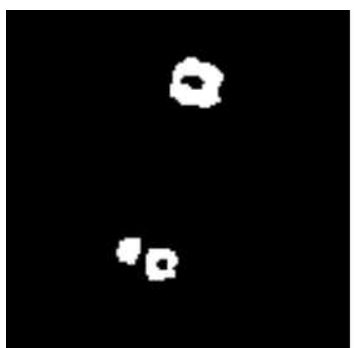

(c) RIS, best threshold

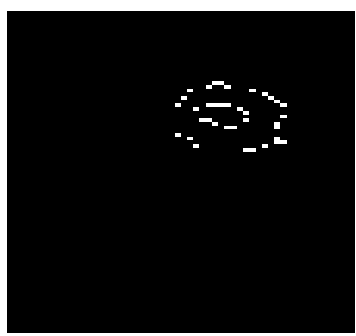

(f) RIS, best threshold

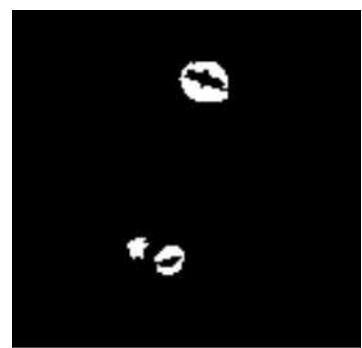

(d) PIS, best threshold

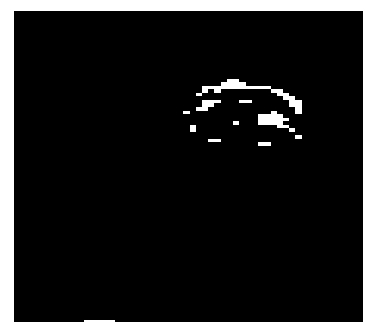

(g) PIS, best threshold

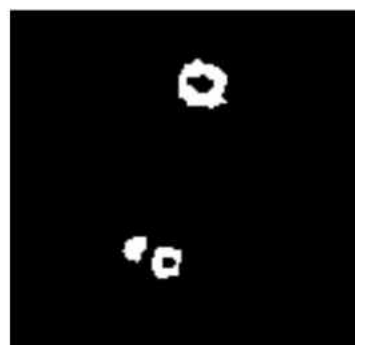

(e) HS, Otsu's threshold

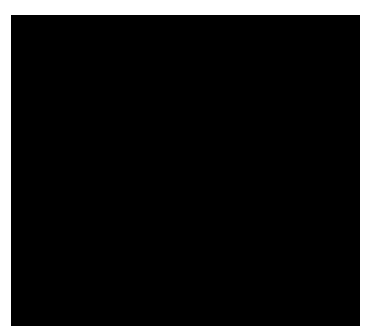

(h) HS, Otsu's threshold space: segmentation results (second Fig. 4. Visual comparison of MAS
row) and error image (third row).

results in reconstructed image

filtering. However, more complicated methods such as Graph Cut can be employed. When the Otsu's threshold is used for RIS and PIS, the entire teeth are decided as metal. Thus we have shown the best segmentation results achievable for RIS and PIS by manually searching for the optimal threshold for each case. In order to clearly compare the segmentation results, we have provided error images in the third row of Fig. 4 that are obtained by differencing between the ground truth and each segmentation result. They indicate that the proposed hybrid segmentation method in Fig. 4 (h) gives the best result. The results for RIS (Fig. 4 (f)) and PIS (Fig. $4(\mathrm{~g}))$ using the best thresholds show that near-metal area is segmented as metal due to artifacts near metal.

\section{CONCLUSIONS}

This research has presented a novel feature space useful for MAS in X-ray CT images. We have shown how to make use of the RNA idea developed for projection image inpainting to create the feature for MAS. It facilitates the segmentation problem by distributing metal pixels and non-metal pixels near metal with opposite signs, and is ex ${ }^{-}$ pected to significantly contribute to the solution of metal area segmentation in the presence of metal artifacts. We are investigating into its extension to the case cone-beam CT images.

\section{REFERENCES}

[1] Y. Kim, S. Yoon, and J. Yi, "Effective Sino- 
gram-inpainting for Metal Artifacts Reduction in $\mathrm{X}-$ ray CT Images," IEEE International Conference on Image Processing, pp. 597-600, 2010.

[2] N. Otsu, "A Threshold Selection Method from Gray-level Histograms," IEEE Transactions on Systems, Man, and Cybernetics, Vol. 9, No. 1, pp. 62-66, 1979.

[3] G. Jang, H. Park, S. Lee, D. Kim, and M. Im, "An Effective Extraction Algorithm of Pulmonary Regions Using Intensity-level Maps in Chest X-ray Images," Journal of Korea Multimedia Society, Vol. 13, No. 7, pp. 1062-1075, 2010.

[4] Wouter J. H. Veldkamp, Raoul M. S. Joemai, Aart J. van der Molen, and Geleijns J., "Development and Validation of Segmentation and Interpolation Techniques in Sinograms for Metal Artifact Suppression in CT," Medical Physics, Vol. 37, No. 2, pp. 620-628, 2010.

[5] H. Xue, Y. Xiao, Z. Chen, and Y. Xing, "Metal Artifact Reduction in Dual Energy CT by Sinogram Segmentation Based on Active Contour Model and TV Inpainting," IEEE Nuclear Science Symposium Conference Record, pp. 904-908, 2009.
[6] J. Gu, L. Zhang, G. Yu, Y. Xing, and Z. Chen, "X-ray CT Metal Artifacts Reduction Through Curvature Based Sinogram Inpainting," Journal of X-Ray Science and Technology, Vol. 14, No. 2, pp. 73-82, 2006.

[ 7 ] M. Oehler and T. M. Buzug, "The $\lambda$-MLEM Algorithm: An Iterative Reconstruction Technique for Metal Artifact Reduction in CT," Advances in Medical Engineering, Vol. 114, No. 1, pp. 42-47, 2007.

[8] H. Akhoondali, R. A. Zoroofi, and G. Shirani, "Rapid Automatic Segmentation and Visualization of Teeth in CT-scan Data," Journal of Applied Sciences, Vol. 9, No. 11, pp. 20312044, 2009.

[9] Y. Zhang et al, "Reducing Metal Artifacts in Cone-beam CT Images by Preprocessing Projection Data," International Journal of Radiation Oncology, Biology, Vol. 67, No. 3, pp. 924-32, 2007.

[10 ] J. H. Hubbell and S. M. Seltzer, "Tables of $\mathrm{X}$-Ray Mass Attenuation Coefficients from 1 $\mathrm{keV}$ to $20 \mathrm{MeV}$ for Elements $Z=1$ to 92 and 48 Additional Substances of Dosimetric Interest and Mass Energy-Absorption Coefficients. Available," http://physics.nist.gov/PhysRef Data/XrayMassCoef/cover.html, 1996. 


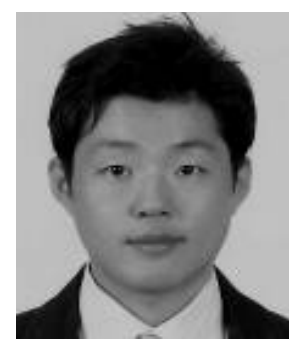

\section{Youngshin Kim}

received the B.S. and Integrated degrees of M. S. and Ph. D. in Electrical and Computer Engineering from Sungkyunkwan University, Korea, in 1999 and 2012, respectively. He is currently a senior researcher at Hyundai Mobis Research Institute, Korea. His primary research interests include medical image processing and object recognition based on the depth image using LIDAR (LIght Detection And Ranging) sensor.

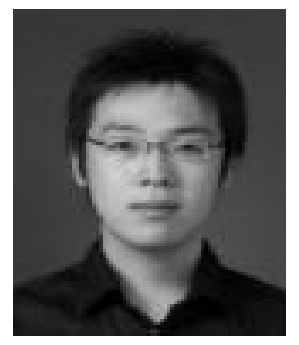

\section{Hyukjoon Kwon}

received the B.S. in Computer Engineering from Seoul National University of Technology and M. S. degrees in Electrical and Computer Engineering from Sungkyunkwan University, Korea, in 2008 and 2012, respectively. He is now a researcher at Huvitz, Korea. His current research interests include Signal processing, pattern recognition in medical image using Optical coherence tomography (OCT).

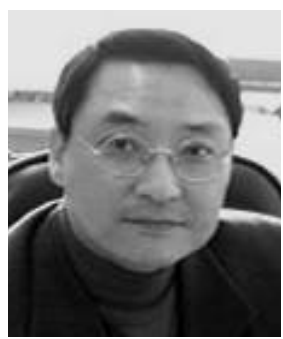

\section{Joongkyu Kim}

received the B.S. and M. S. degrees in Electronic Engineering from Seoul National University, Korea, in 1980 and 1982, respectively, and the Ph.D. degree in Electrical Engineering and Computer Science from the University of Michigan, Ann Arbor, in 1989. He is currently a professor of Electronic and Electrical Engineering, College of Information and Communication Engineering, Sungkyunkwan University, Korea. His research interests lie in the areas of signal processing: signal detection and estimation, active noise canceling, biomedical signal and image processing, and applications of time frequency distribution for nonstationary signal analysis.

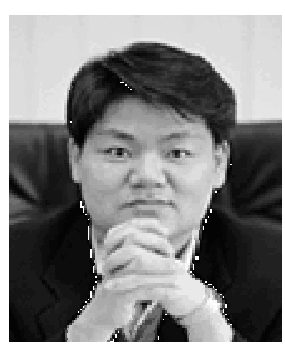

\section{Juneho Yi}

received the B.S. degree from the Seoul National University, Seoul, Korea, in 1985, the M. S. degree from the Pennsylvania State University, University Park, PA, in 1987, and the Ph.D. degree from Purdue University in 1994, all in Electrical and Computer Engineering. He is currently a professor of Electronic and Electrical Engineering, College of Information and Communication Engineering, Sungkyunkwan University, Korea. His research interests are broadly in the areas of vision-based computer interaction. 